Подписана в печать 15.06.2020

\title{
КВАНТОВАЯ МОДИФИКАЦИЯ АЛГОРИТМА ПРЕСНОВОДНЫХ ГИДР ДЛЯ РЕШЕНИЯ ЗАДАЧИ ОПТИМИЗАЦИИ
}

\author{
(C) 2020 С. А. Королев ${ }^{1}$, Д. В. Майков ${ }^{\bowtie 2}$ \\ ${ }^{1}$ Ижевский государственный технический университет имени М. Т. Калашникова \\ ул. Студенческая, 7, 426069 Ижевск, Российская Федерация \\ ${ }^{2}$ Ижевский торгово-экономический техникум \\ ул. Ворочилова, 20а, 426000 Ижевск, Российская Федерачия
}

\begin{abstract}
Аннотация. Одним из этапов решения ряда задач математического моделирования является поиск точки, в которой некоторая функция нескольких переменных достигает наибольшего или наименьшего значения. Эта функция, как правило, имеет высокую размерность и множество локальных экстремумов. Подобная задача успешно решается популяционными алгоритмами оптимизации, например, алгоритмом роя частиц, алгоритмом пресноводных гидр и другими. Целью работы являлось совершенствование алгоритма пресноводных гидр (Н-алгоритма) с учетом представлений квантовой механики. Разработанные квантовые модификации данного алгоритма основаны на методе анализа иерархий $(\mathrm{QH}-\mathrm{AHP})$ и байесовском подходе (QH-B). Скорость сходимости квантовых модификаций выше, чем у исходного Н-алгоритма в связи с тем, что положение особей определяется напрямую, не оперируя их скоростями (в соответствии с представлениями квантовой механики). Вследствие этого, в течение одной итерации особи могут перемещаться на значительное расстояние, в результате чего увеличивается охват пространства поиска. Кроме того, данный подход позволяет особям успешнее преодолевать области притяжения локальных экстремумов, что препятствует преждевременной сходимости алгоритма. Найдены оптимальные значения параметров разработанных алгоритмов в результате решения задачи метаоптимизации. Сравнение скорости сходимости предложенных алгоритмов оптимизации выполнялось на примере различных многоэкстремальных тестовых функций: Розенброка, Дэвиса, Экли, Растригина. Предложенные квантовые модификации Н-алгоритма (QH-AНP и QН-B) для различных тестовых функций показали различную, но в среднем одинаковую скорость сходимости. Скорость сходимости квантовых модификаций алгоритмов для различных тестовых функций выше, чем у исходного алгоритма пресноводных гидр и алгоритма роя частиц. Разработанные алгоритмы решения задачи оптимизации могут быть использованы при обучении нейронных сетей, при математическом моделировании процессов и систем в различных предметных областях на этапе идентификации параметров модели, оптимизации характеристик и построения оптимального управления такими системами и т. д.
\end{abstract}

Ключевые слова: задача оптимизации, алгоритм роя частиц, квантовый алгоритм пресноводных гидр, метод анализа иерархий, байесовский подход, задача метаоптимизации.

\section{ВВЕДЕНИЕ}

При решении ряда задач математического моделирования возникает необходимость нахождения точки экстремума некоторой функции многих переменных (целевой функции).

Майков Дмитрий Владимирович e-mail: MaykovD@yandex.ru
Такими задачами являются, например, оптимизация параметров технических и экономических систем $[1,2,3]$, минимизация функции ошибки при обучении нейронных сетей [4], решение задачи идентификации параметров модели по экспериментальным данным [5], построение оптимального управления для динамических систем [6] и др.

Контент доступен под лицензией Creative Commons Attribution 4.0 License.

The content is available under Creative Commons Attribution 4.0 License. 


\section{С. А. Королев, Д. В. Майков}

Задача условной многомерной оптимизации, возникающая в подобных примерах, в общем случае (для определенности рассмотрим задачу минимизации) имеет вид:

$$
\mathbf{x}^{\text {opt }}=\arg \min _{\mathbf{x} \in D} f(\mathbf{x}) .
$$

Требуется найти такой вектор переменных $\mathbf{x}$, при котором функция $f(\mathbf{x})$ достигает глобального экстремума в некоторой замкнутой $n$-мерной области $D$. При этом функция $f(\mathbf{x})$ может иметь множество локальных экстремумов и не выражаться аналитически.

Для решения этой задачи успешно применяются популяционные алгоритмы оптимизации, основанные на механизмах, заимствованных из живой природы: алгоритм роя частиц, летучих мышей и др. [7]. Эти алгоритмы работают сразу с несколькими допустимыми векторами из области $D$, которые называются особями, агентами или частицами. Совокупность особей образует популяцию. Особь с наилучшим значением целевой функции называется лидером.

Примером популяционного алгоритма является алгоритм роя частиц (Particle Swarm Optimization, PSO), в основу которого положены модели движения толпы людей, стада зверей, стаи птиц [7]. Координаты каждой частицы в пространстве поиска в текущий момент времени определяются через ее координаты в предыдущий момент времени и скорость движения. Скорость частицы складывается из нескольких компонент: инерционной (служащей для предотвращения резких изменений скорости частицы), когнитивной (отражающей память частицы о ее наилучшем положении) и социальной (представляющей собой память всего роя о наилучшем положении его частиц).

Одним из популяционных алгоритмов является предложенный авторами алгоритм пресноводных гидр (Н-алгоритм) [8]. В нем для определения направления движения особей используется метод анализа иерархий. При этом за основу принимаются три направления: текущее, к лидеру и случайное. В алгоритм заложены механизмы, подобные поведению пресноводных гидр. Так, при наступлении неблагоприятных условий среды гидра погибает, выпуская в воду яйцо, переносимое с током воды, из которого вырастает новая особь гидры. Этот факт формализован в алгоритме с помощью оператора переноса, когда новые координаты особи задаются случайным образом по определенному правилу.

Популяционные алгоритмы интенсивно развиваются. Одним из направлений их совершенствования является применение представлений квантовой механики. Известны квантовые модификации алгоритма роя частиц [9], летучих мышей [10], колонии искусственных пчел [11], генетических алгоритмов [12] и др. Целью данной работы является разработка квантовой модификации алгоритма пресноводных гидр.

Одним из важнейших уравнений квантовой механики является уравнение Шрёдингеpa [9]:

$$
i \hbar \frac{\partial \Psi(\mathbf{x}, t)}{\partial t}=H \Psi(\mathbf{x}, t),
$$

где $i-$ мнимая единица, $\hbar=\frac{h}{2 \pi}(h-$ постоянная Планка), $H$ - гамильтониан.

Решением этого уравнения служит волновая функция $\Psi(\mathbf{x}, t)$, квадрат модуля которой равен вероятности нахождения квантовой частицы в малой окрестности точки $\mathbf{x}$ в момент времени $t$. Таким образом, в отличие от классической механики, в которой на основе текущих координат частицы и ее скорости можно однозначно определить положение этой частицы в любой момент времени, в квантовой механике определяется лишь вероятность обнаружения частицы в окрестности заданной точки пространства. Кроме того, согласно принципа неопределенности Гейзенберга, нельзя одновременно определить точные значения координат и скорости (либо импульса) квантовой частицы.

Если частица движется в одномерной потенциальной яме с центром в точке $q$, то выражение для координаты частицы, полученное путем решения данного уравнения с помощью метода Монте-Карло, выглядит следующим образом:

$$
x=q \pm \frac{\hbar^{2}}{2 m} \ln \frac{1}{\alpha},
$$


где $m$ - масса частицы, $\alpha-$ случайная величина, равномерно распределенная на интервале $(0,1)$.

В классических популяционных алгоритмах для нахождения нового положения особи часто используется ее скорость (алгоритм роя частиц, алгоритм летучих мышей, Н-алгоритм), а в их квантовых модификациях координаты частиц определяются непосредственно с некоторой вероятностью. Это позволяет увеличить охват пространства поиска, а также повысить устойчивость алгоритма к попаданию в локальный экстремум и преждевременной сходимости.

\section{МЕТОДЫ ИССЛЕДОВАНИЯ}

\section{Квантовый алгоритм пресноводных гидр на основе метода анализа иерархий (QH-АНР-алгоритм)}

Для работы популяционных алгоритмов случайным образом создается популяция из $S$ особей $\mathbf{x}_{j}(j=\overline{1, S})$, являющихся возможными решениями. При этом координаты особей $x_{i j}(i=\overline{1, n})$ принимают случайные значения из заданного промежутка $\left[x_{i}^{\min }, x_{i}^{\max }\right]$.

Направление движения особи определяется с помощью метода анализа иерархий (Analytic Hierarchy Process, AHР). Этот метод позволяет выбирать решение среди $p$ возможных вариантов [13]. Предпочтения в пользу того или иного варианта выражаются матрицей парных сравнений $A$. Каждый элемент $a_{i j}$ этой матрицы принимает значения из промежутка $[0,1,10]$ и показывает, насколько $i$-й вариант лучше $j$-го $(i, j=\overline{1, p})$, причем $a_{i j}=\frac{1}{a_{j i}}$ и $a_{i i}=1$.

Нормируя элементы матрицы $A$ по правилу $b_{i j}=\frac{a_{i j}}{\sum_{i=1}^{p} a_{i j}}$, формируется матрица $B$.

Степень предпочтения $i$-го варианта выражается его весовым коэффициентом, равным среднему значению элементов $i$-й строки матрицы $B$ :

$$
w_{i}=\frac{1}{p} \sum_{j=1}^{p} b_{i j} .
$$

В предлагаемом методе выбираются следующие варианты направления движения особи: к среднему вектору $\mathbf{C}$ лучших положений особей популяции, к лидеру и случайное.

Вектор $\mathbf{C}^{k}$ представляет собой среднюю лучшую позицию всех особей:

$$
C_{i}^{k}=\frac{1}{S} \sum_{j=1}^{S} x_{i j}^{*}
$$

где $x_{i j}^{*}$ - координаты $j$-й особи с наилучшим значением целевой функции за все время поиска.

Начальные значения элементов матрицы $A$ представлены в табл. 1, им соответствуют хорошие показатели скорости сходимости алгоритма.

Таблица 1. Начальные значения элементов матрии, парных сравнений

[Table 1. Initial values of the elements of the matrices of pairwise comparisons]

\begin{tabular}{|c|c|c|c|}
\hline $\begin{array}{c}\text { Направле- } \\
\text { ние }\end{array}$ & $\begin{array}{c}\text { К средней } \\
\text { пучшей } \\
\text { позиции }\end{array}$ & К лидеру & Случайное \\
\hline $\begin{array}{c}\text { К средней } \\
\text { пучшей } \\
\text { позиции }\end{array}$ & 1 & $\frac{1}{3}$ & $\frac{1}{6}$ \\
\hline К лидеру & 3 & 1 & $\frac{1}{4}$ \\
\hline Случайное & 6 & 4 & 1 \\
\hline
\end{tabular}

На основе этой матрицы по формуле (1) вычисляются приоритеты направлений и выполняется шаг в направлении с большим приоритетом. Движение к среднему вектору $\mathbf{C}$ лучших положений особей популяции выполняется по формулам [9]:

$$
x_{i j}^{k+1}=\left\{\begin{array}{l}
p_{i j}^{k}+\lambda^{k} \cdot\left|C_{i}^{k}-x_{i j}^{k}\right| \cdot \ln \frac{1}{\alpha_{i j}^{k}}, \beta_{i j}^{k} \geq 0,5, \\
p_{i j}^{k}-\lambda^{k} \cdot\left|C_{i}^{k}-x_{i j}^{k}\right| \cdot \ln \frac{1}{\alpha_{i j}^{k}}, \beta_{i j}^{k}<0,5,
\end{array}\right.
$$

где случайные величины $\alpha_{i j}$ и $\beta_{i j}$ принадлежат интервалу $(0,1)$.

Координаты аттрактора каждой особи определяются следующим образом:

$$
p_{i j}^{k}=\varphi_{i j}^{k} x_{i j}^{*}+\left(1-\varphi_{i j}^{k}\right) x_{i}^{* *},
$$

где $x_{i}^{* *}$ - координаты лучшего решения (лидера) за все время поиска; случайные величины $\varphi_{i j}$ принимают значения из диапазона $[0,1]$. 


\section{С. А. Королев, Д. В. Майков}

Значение параметра $\lambda$ определяется выражением:

$$
\lambda^{k}=\lambda_{1}+\frac{\lambda_{0}-\lambda_{1}}{k},
$$

где $\lambda_{0}-$ его начальное значение (при $k=1$ ), а к значению $\lambda_{1}$ данный параметр стремится при $k \rightarrow \infty$. В общем случае параметр $\lambda$ является векторным. При этом $i$-я координата векторов $\lambda_{0}$ и $\lambda_{1}$ может определяться с помощью соотношения вида $w \cdot\left(x_{i}^{\max }-x_{i}^{\min }\right)$ с коэффициентом $w \in(0,1)$.

Для каждой координаты особи задаются две случайные величины $\gamma_{i j}$ и $\chi_{i j}$, принадлежащие интервалу $(0,1)$. На их основе вычисляются две независимые случайные величины [14]:

$$
\begin{aligned}
& \xi_{i j}=\sqrt{-2 \ln \gamma_{i j}} \cdot \cos 2 \pi \chi_{i j}, \\
& \eta_{i j}=\sqrt{-2 \ln \gamma_{i j}} \cdot \sin 2 \pi \chi_{i j},
\end{aligned}
$$

закон распределения которых близок к стандартному нормальному $\mathrm{N}(0,1)$. Также генерируются равномерно распределенные на промежутке $[0,1]$ случайные величины $\alpha_{i j}$ и $\beta_{i j}$.

Переход в направлении к лидеру осуществляется согласно соотношения:

$$
x_{i j}^{k+1}=x_{i j}^{k}+\lambda^{k} \cdot \xi_{i j}^{k} \cdot\left(x_{i}^{* *}-x_{i j}^{k}\right) \cdot \alpha_{i j}^{k} .
$$

Перемещение особи в случайном направлении реализуется следующим образом.

$$
x_{i j}^{k+1}=\left\{\begin{array}{l}
x_{i j}^{*}+\lambda^{k} \cdot \eta_{i j}^{k}, \beta_{i j}^{k} \geq 0,5, \\
x_{i j}^{*}-\lambda^{k} \cdot \eta_{i j}^{k}, \beta_{i j}^{k}<0,5 .
\end{array}\right.
$$

Для увеличения охвата пространства поиска и предотвращения преждевременной сходимости алгоритма к локальному экстремуму применяется оператор переноса. Он выполняется всякий ра3, когда количество итераций $\tau_{j}$, в течение которых целевая функция $j$-й особи не улучшалась, становится равным пороговому значению $\tau_{\max }$. При этом для каждой координаты особи $\mathbf{x}_{j}^{k}$ генерируются случайные числа $\alpha_{i j}$ и $\beta_{i j}$ из промежутка $[0,1]$ и эта особь заменяется новой особью с координатами:

$$
x_{i j}^{k+1}=\left\{\begin{array}{l}
x_{i j}^{k}+\alpha_{i j} \cdot\left(x_{i}^{\max }-x_{i j}^{k}\right) \cdot g(k), \beta_{i j} \geq 0,5 \\
x_{i j}^{k}-\alpha_{i j} \cdot\left(x_{i j}^{k}-x_{i}^{\min }\right) \cdot g(k), \beta_{i j}<0,5 .
\end{array}\right.
$$

Функция $g(k)$ выбирается так, чтобы она принимала значения из промежутка $[0,1]$. В этом случае и значения $x_{i j}^{k+1}$ остаются внутри промежутка $[0,1]$. В данной работе использована функция вида:

$$
g(q)=\frac{1}{2} \cdot\left(1-\frac{q}{\mu+|q|}\right), q=k-\frac{K}{2} .
$$

Установлено, что хорошим показателям сходимости алгоритма соответствует значение параметра $\mu=88$. Параметр $K$ равен характерному количеству итераций, по достижении которого целевая функция перестает существенно изменяться. В данной работе принималось $K=1000$.

Для популяционных алгоритмов существуют различные критерии окончания поиска. В данной работе было выбрано следующее условие [11]:

$$
\chi=\max _{q}\left|\frac{f^{k}-f^{k-q}}{f^{k}}\right|<10^{-4},
$$

где $q=1, \ldots, \min (k, \omega)$; значение параметра $\omega$ принимается равным 100 итерациям.

Разработанный алгоритм состоит в следующем:

I. Создать начальную популяцию. Для каждой $j$-й особи задать матрицу парных сравнений $A_{j}$ (см. табл. 1), определить значение целевой функции, положить число итераций $\tau_{j}$, в течение которых целевая функция не улучшалась, равным нулю. Задать номер итерации $k=1$. Найти лидера.

II. По формуле (2) определить средний вектор $\mathbf{C}$ лучших положений особей популяции и найти значение параметра $\lambda$ с помощью выражения (4).

III. Для каждой $j$-й особи выполнить следующее:

1. Найти вектор приоритетов $\mathbf{w}(1)$.

2. Выполнить шаг в направлении с наибольшим приоритетом $p_{\max }=\arg \max _{p} w_{p}$ (формулы (3), (5), (6)). Если он одинаков у нескольких направлений, то выбрать любое из них.

3. Вычислить значение целевой функции. Если оно улучшилось, то в строке $i=p_{\max }$ матрицы $A$ увеличить элементы $a_{i j}$ (например, на 1 , если $a_{i j}>1$, и на 0,1 , если $\left.0<a_{i j}<1\right)$. Если при этом значение $a_{i j}$ оказывается вне диапа- 
зона $[0,1,10]$, то положить его равным ближайшему граничному значению. Положить $\tau_{j}$ равным нулю. Обновить лучшее положение особи $\mathbf{x}_{j}^{*}$, если это возможно.

4. Если значение целевой функции ухудшается, то уменьшить соответствующие элементы матрицы $A$. Увеличить $\tau_{j}$ на единицу.

5. Пересчитать элементы, симметричные тем, которые изменились: $a_{j i}=\frac{1}{a_{i j}}$.

6. Если $\tau_{j}=\tau_{\max }$, то применить оператор переноса (7), рассчитать значение целевой функции в новой точке и вернуть элементам матрицы $A_{j}$ исходные значения (см. табл. 1 ).

IV. Найти лидера и проверить выполнение критерия останова (8). В случае выполнения завершить поиск. Положить $k=k+1$ и перейти к шагу II (следующей итерации алгоритма).

\section{Квантовый алгоритм пресноводных гидр на основе байесовского подхода (QH-В-алгоритм)}

Для выбора направления движения особи, помимо метода анализа иерархий, могут использоваться и другие методы теории принятия решений, в частности, байесовский подход [15]. При этом рассматриваются следующие случайные события (гипотезы):

$H_{1}$ - особь перемещается к среднему вектору $\mathbf{C}$ лучших положений всех особей популяции;

$H_{2}$ - особь движется в направлении к лидеру;

$H_{3}$ - перемещение особи осуществляется случайным образом.

В результате перемещения особи в том или ином направлении появляются следующие свидетельства:

$A-$ значение целевой функции особи улучшилось;

$\bar{A}$ - значение целевой функции особи ухудшилось.

На основе события $A$ делается вывод о правильности той или иной гипотезы. Предполагается, что события $H_{1}, H_{2}, H_{3}$ образуют полную группу событий. При использовании байесовского подхода исходные априорные вероятности гипотез $P\left(H_{1}\right), P\left(H_{2}\right), P\left(H_{3}\right)$ последовательно пересчитываются на основе поступающих свидетельств $A$ или $\bar{A}$. Согласно формулы Байеса, апостериорные оценки этих вероятностей равны:

1) в случае наступления события $A$ :

$$
\begin{gathered}
P\left(H_{r} \mid A\right)=\frac{P\left(H_{r}\right) \cdot P\left(A \mid H_{r}\right)}{\sum_{s=1}^{3} P\left(H_{s}\right) \cdot P\left(A \mid H_{s}\right)}, \\
r \in\{1,2,3\} ;
\end{gathered}
$$

2) в случае наступления события $\bar{A}$ :

$$
\begin{aligned}
& P\left(H_{r} \mid \bar{A}\right)= \frac{P\left(H_{r}\right) \cdot P\left(\bar{A} \mid H_{r}\right)}{\sum_{s=1}^{3} P\left(H_{s}\right) \cdot P\left(\bar{A} \mid H_{s}\right)}, \\
& r \in\{1,2,3\} .
\end{aligned}
$$

Для определения вероятностей рассматриваемых событий необходимо учитывать, что в начале поиска лучшее решение (лидер), как правило, далеко от оптимального, поэтому предпочтительно движение особи в случайном направлении. На завершающих этапах поиска вероятность движения в случайном направлении должна быть наименьшей, т. к. необходимо уточнить найденное решение.

Зависимость условных вероятностей $P\left(A \mid H_{r}\right)$ от номера итерации $k$ определялась выражением:

$$
P\left(A \mid H_{r}\right)=w_{r}+\frac{u_{r}-w_{r}}{k},
$$

где $u_{r}$ представляет собой начальное значение вероятности $\left(P\left(A \mid H_{r}\right)=u_{r}\right.$ при $\left.k=1\right)$, а к значению $w_{r}$ эта вероятность стремится при $k \rightarrow \infty$.

Разработанный алгоритм имеет следующий вид:

I. Создать начальную популяцию. Для каждой особи задать вероятности $P\left(H_{1}\right)$, $P\left(H_{2}\right), P\left(H_{3}\right)$, определить значение целевой функции и положить количество итераций $\tau_{j}$, в течение которых не происходило улучшение значения целевой функции, равным нулю. Найти лидера. Положить $k=1$.

II. По формуле (2) определить средний вектор $\mathbf{C}$ лучших положений особей популяции. Для всех особей вычислить вероятности $P\left(A \mid H_{1}\right), P\left(A \mid H_{2}\right), P\left(A \mid H_{3}\right)$ как функции от номера итерации и значение параметра $\lambda \mathrm{c}$ помощью выражения (4). 
III. Для каждой $j$-й особи выполнить следующее:

1. Осуществить перемещение по формулам (3), (5), (6) в направлении с наибольшей вероятностью $P\left(H_{1}\right), P\left(H_{2}\right), P\left(H_{3}\right)$. Если эти вероятности равны для нескольких направлений, то выбрать любое из них случайным образом.

2. Вычислить значение целевой функции. Если оно улучшилось (наступило событие $A$ ), то вычислить вероятности $P\left(H_{r}\right)$, считая, что они равны апостериорным вероятностям формулы (9). Положить $\tau_{j}$ равным нулю. Обновить лучшее положение особи $\mathbf{x}_{j}^{*}$, если это возможно.

3. Если значение целевой функции ухудшилось, то пересчитать вероятности $P\left(H_{r}\right)$ по формуле (10). Увеличить $\tau_{j}$ на единицу. Если $\tau_{j}=\tau_{\max }$, то применить оператор переноса (7), рассчитать значение целевой функции в новой точке и вернуть вероятностям $P\left(H_{r}\right)$ исходные значения $\left(P\left(H_{1}\right)=0,23\right.$, $\left.P\left(H_{2}\right)=0,18, P\left(H_{3}\right)=0,71\right)$.

IV. Найти лидера и проверить выполнение критерия останова (8). В случае выполнения завершить поиск, иначе положить $k=k+1$ и перейти к шагу II (следующей итерации алгоритма).

\section{РЕЗУЛЬТАТЫ ИССЛЕДОВАНИЯ И ИХ ОБСУЖДЕНИЕ}

Сравнение скорости сходимости рассматриваемых методов выполнялось на примере следующих тестовых функций [7]:

1) $f_{1}(\mathbf{x})=\sum_{i=1}^{n-1}\left(100 \cdot\left(x_{i+1}-x_{i}^{2}\right)^{2}+\left(1-x_{i}\right)^{2}\right)-$ функция Розенброка. Оптимальное решение $x_{i}^{o p t}=1, i=\overline{1, n}, f_{1}\left(\mathbf{x}^{o p t}\right)=0$.

2) $f_{2}(\mathbf{x})=\sum_{i=1}^{n-1}\left(x_{i+1}^{2}+x_{i}^{2}\right)^{0,25} \times$

$\times\left(\sin ^{2}\left(50\left(x_{i+1}^{2}+x_{i}^{2}\right)^{0,1}\right)+1\right)-$ функция Дэвиса. Оптимальное решение $x_{i}^{\text {opt }}=0, i=\overline{1, n}$, $f_{2}\left(\mathbf{x}^{\text {opt }}\right)=0$.

3) $f_{3}(\mathbf{x})=-20 \exp \left(-0,2 \cdot \sqrt{\frac{1}{n} \sum_{i=1}^{n} x_{i}^{2}}\right)-$ $-\exp \left(\frac{1}{n} \sum_{i=1}^{n} \cos \left(2 \pi x_{i}\right)\right)+\exp (1)+20-$ функ- ция Экли. Оптимальное решение $x_{i}^{o p t}=0$, $i=\overline{1, n}, f_{3}\left(\mathbf{x}^{\text {opt }}\right)=0$.
4) $f_{4}(\mathbf{x})=\sum_{i=1}^{n} 10\left(1-\cos \left(2 \pi x_{i}\right)\right)+x_{i}^{2}$

функция Растригина. Оптимальное решение $x_{i}^{o p t}=0, i=\overline{1, n}, f_{4}\left(\mathbf{x}^{o p t}\right)=0$.

В качестве диапазона изменения всех координат $x_{i}$ был выбран промежуток $[-10,10]$. Рассматривались функции от $n=10$ переменных. Количество особей в популяции принималось равным 100.

Для достижения наибольшей эффективности работы популяционных алгоритмов необходимо решить задачу метаоптимизации, т. е. найти оптимальные значения параметров алгоритма для конкретной целевой функции [10]. Такими параметрами QH-AНР-алгоритма служат $a_{i j} \in[1, \ldots, 10], \quad i<j, \quad i, j=1,2,3$, $S \in[10,200], \quad \lambda_{0} \in(0,10), \quad \lambda_{1} \in(0,10)$, $\mu \in[10,200], \quad \tau_{\max } \in[1,50]$. Для QН-В-алгоритма такими параметрами являются $P\left(H_{1}\right)$, $P\left(H_{2}\right), P\left(H_{3}\right), u_{1}, u_{2}, u_{3}, w_{1}, w_{2}, w_{3}, S, \lambda_{0}, \lambda_{1}, \mu$, $\tau_{\max }$. При этом $P\left(H_{r}\right) \in[0,1], \quad u_{r} \in[0,1]$, $w_{r} \in[0,1], r=\overline{1,3}$.

В качестве зависящей от этих параметров целевой метафункции $\psi$ принималось среднее значение целевой функции $f_{q}(q=\overline{1,4})$, достигаемое за 10 запусков алгоритма, причем длительность каждого запуска ограничивалась 100 секундами. Для получения результатов, оптимальных в среднем для всех тестовых функций $f_{q}$, на каждой итерации метаалгоритма она выбиралась случайным образом. В качестве метаалгоритма использован алгоритм роя частиц, состоящий из 100 агентов.

На рис. 1 показаны графики зависимости скорости сходимости алгоритмов оптимизации (значений целевой метафункции $\psi$ ) от значений настраиваемых параметров алгоритма.

В результате решения задачи метаоптимизации для QН-АНР-алгоритма получены следующие оценки параметров: $a_{21}=3, a_{31}=6$, $a_{32}=4, \quad S=87, \quad \lambda_{0}=0,54, \quad \lambda_{1}=0,13, \quad \mu=88$, $\tau_{\max }=21$. Для QH-В-алгоритма оценки параметров равны $P\left(H_{1}\right)=0,23, P\left(H_{2}\right)=0,18$, $P\left(H_{3}\right)=0,71, \quad u_{1}=0,12, \quad u_{2}=0,16, \quad u_{3}=0,72$, $w_{1}=0,48, w_{2}=0,52, w_{3}=0,85, S=78, \lambda_{0}=0,42$, $\lambda_{1}=0,13, \mu=94, \tau_{\max }=17$. 


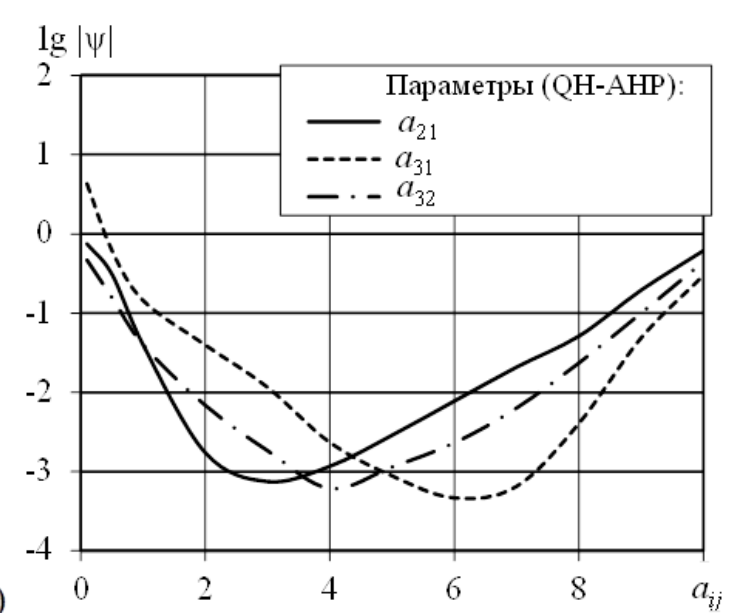

a)

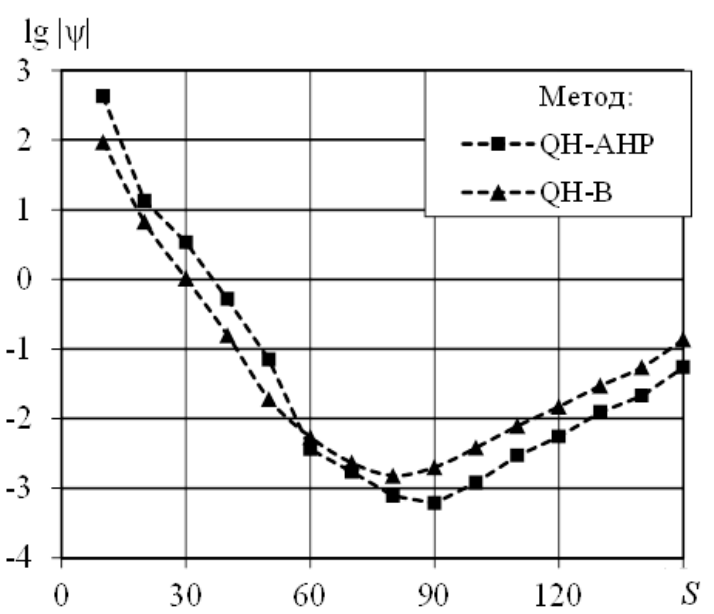

б)

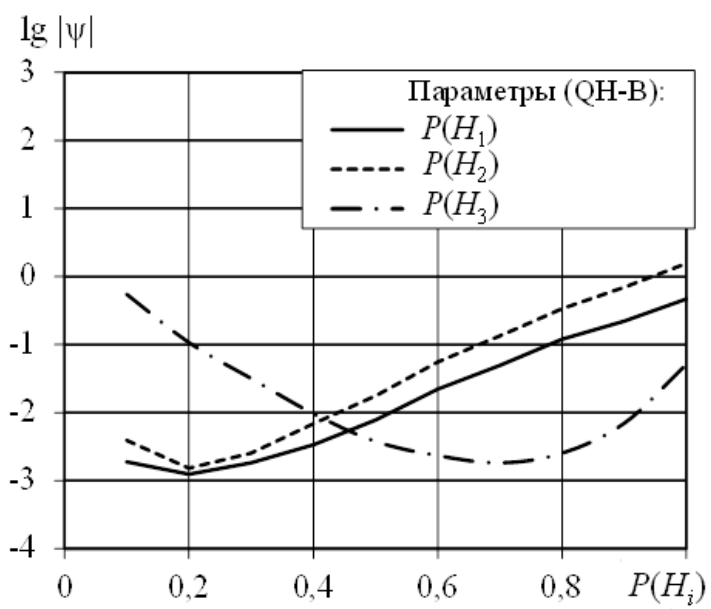

$\lg |\psi|$

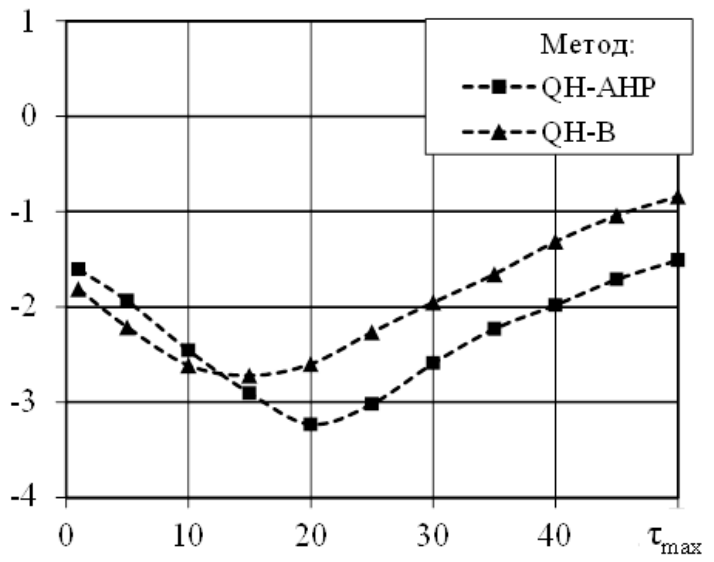

Рис. 1. Грабики зависимости скорости сходимости алгоритмов от значений параметров:

a) QH-AHP-алгоритма от $a_{i j}$; б) QH-B-алгоритма от $P\left(H_{r}\right)$;

в) QH-AHP и QH-B алгоритмов от $S$; г) QH-AHP и QH-B алгоритмов от $\tau_{\max }$

[Fig. 1. Graphs of the dependence of the convergence rate of algorithms on parameter values:

a) QH-AHP-algorithm depends on the $a_{i j}$; b) QH-B-algorithm depends on the $P\left(H_{r}\right)$;

c) $\mathrm{QH}-\mathrm{AHP}$ and $\mathrm{QH}-\mathrm{B}$ algorithm depends on the $S ; d) \mathrm{QH}-\mathrm{AHP}$ and $\mathrm{QH}-\mathrm{B}$ algorithm depends on the $\left.\tau_{\max }\right]$

Полученные оптимальные значения параметров использовались в дальнейшем при исследовании скорости сходимости рассматриваемых алгоритмов оптимизации на тестовых функциях. Графики скорости сходимости для тестовых функций (средние значения целевой функции лидера за 10 запусков алгоритма) показаны на рис. 2.

Рассматривались следующие алгоритмы оптимизации:

1) $\mathrm{PSO}$ - алгоритм роя частиц;

2) Н - алгоритм пресноводных гидр;

3) QH-AНP - квантовый алгоритм пресноводных гидр на основе метода анализа иерархий;
4) $\mathrm{QH}-\mathrm{B}$ - квантовый алгоритм пресноводных гидр на основе байесовского подхода.

Распределение алгоритмов по скорости сходимости в порядке ее убывания приведено в табл. 2.

Для всех рассматриваемых тестовых функций квантовые модификации алгоритма пресноводных гидр показали более высокую скорость сходимости по сравнению с исходным алгоритмом и алгоритмом роя частиц. В большинстве случаев квантовый алгоритм, основанный на методе анализа иерархий (QH-AНP) показал лучшие результаты, чем аналогичный алгоритм, использующий байесовский подход (QH-B). 


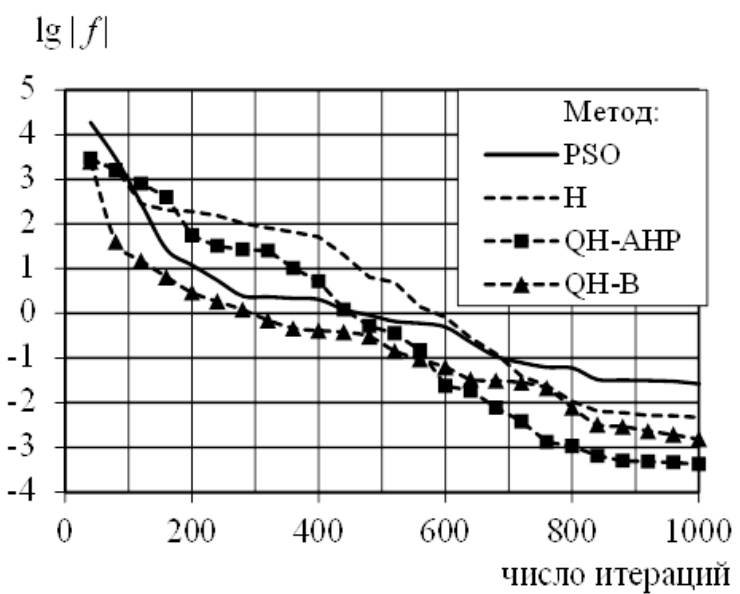

a)

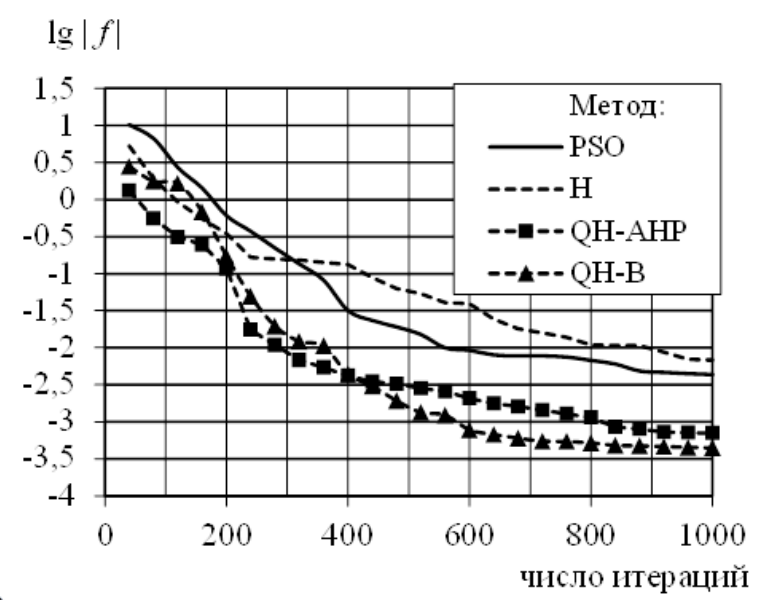

в)

Рис. 2. Графики скорости сходимости на примере тестовых функиий:

а) $f_{1}$ - Розенброка; б) $f_{2}-$ Дэвиса; в) $f_{3}-$ Экли; г) $f_{4}-$ Растригина

[Fig. 2. Graphs of convergence rate for different test functions:

a) $f_{1}$ - Rosenbrock; b) $f_{2}$-Davis; c) $f_{3}$-Ackley; d) $f_{4}$ - Rastrigin]

Таблииа 2. Сравнение скорости сходимости алгоритмов

[Table 2. Comparison of convergence rates of the algorithms]

\begin{tabular}{|l|c|c|c|c|}
\hline \multirow{2}{*}{ Тестовая функция } & \multicolumn{4}{|c|}{ Расположение алгоритмов оптимизации } \\
& 1 & 2 & 3 & 4 \\
\cline { 2 - 5 } & в порядке убывания скорости сходимости \\
\hline$f_{1}-$ Розенброка & QH-АНP & QH-B & H & PSO \\
\hline$f_{2}$ - Дэвиса & QH-AНP & QH-B & PSO & H \\
\hline$f_{3}-$ Экли & QH-B & QH-AHP & PSO & H \\
\hline$f_{4}-$ Растригина & QH-AНP & QH-B & H & PSO \\
\hline
\end{tabular}

\section{ЗАКЛЮЧЕНИЕ}

В работе рассмотрены предложенные авторами модификации алгоритма пресноводных гидр для решения задачи оптимизации. В данных модификациях, разработанных с учетом принципов квантовой механики, выбор направления движения особи осуществляется на основе метода анализа иерархий и байесовского подхода. Найдены оптимальные значения параметров этих алгоритмов путем решения задачи метаоптимизации. 
Квантовая модификация алгоритма пресноводньх гидр...

Проведено тестирование скорости сходимости рассмотренных алгоритмов на примере различных многоэкстремальных функций. Скорость сходимости квантовых модификаций алгоритмов для различных тестовых функций выше, чем у исходного алгоритма пресноводных гидр и алгоритма роя частиц. Это связано с тем, что в квантовых модификациях популяционных алгоритмов положение особей определяется напрямую, без использования понятия ее скорости, так что с некоторой вероятностью особь может находиться в любой точке весьма широкой области. Поэтому в результате выполнения одной итерации особи могут перемещаться на существенное расстояние, вследствие чего для каждой из них возрастает обзор пространства поиска и облегчается преодоление области притяжения в окрестности локальных экстремумов.

\section{СПИСОК ЛИТЕРАТУРЫ}

1. Гуров, E. В. Оптимизация параметров БИХ-фильтров с помощью алгоритма роя частиц / Е. В. Гуров, С. С. Увайсова, В. В. Ковалев, А. С. Увайсова // Инновационные, информационные и коммуникационные технологии. - 2017. - № 1. - С. 552-553.

2. Есиков, О. В. Применение метода роя частиц для решения задачи распределения вычислительных ресурсов в бортовых информационных и управляющих системах по критерию равномерной загрузки / О.В.Есиков, С. М. Цыбин, А. И. Чернышков // Известия Тульского государственного университета. Технические науки. - 2017. - № 9-1. - С. 74-81.

3. Акиншин, О. Н. Особенности решения задачи оптимизации инвестиционного портфеля предприятия методом роя частиц / О. Н. Акиншин, Д. О. Есиков, Н. Ю. Акиншина // Известия Тульского государственного университета. Технические науки. - 2016. - № 5. C. 109-116.

4. Ермаков, Б. С. Оптимизация роем частиц в обучении искусственных нейронных сетей / Б. С. Ермаков // Системный анализ и логистика. - 2017. - № 1 (14). - С. 3-9.

5. Королев, С. А. Идентификация математической модели метаногенеза и исследова- ние различных режимов метаногенеза в мезофильной среде / С. А. Королев, Д. В. Майков // Компьютерные исследования и моделирование. - 2012. - Т. 4, № 1. - С. 131-141. DOI: 10.20537/2076-7633-2012-4-1-131-141

6. Королев, С. А. Решение задачи оптимального управления процессом метаногенеза на основе принципа максимума Понтрягина / С. А. Королев, Д. В. Майков // Компьютерные исследования и моделирование. - 2020. T. 12, № 2. - C. 357-367. DOI: 10.20537/20767633-2020-12-2-357-367.

7. Карпенко, А. П. Современные алгоритмы поисковой оптимизации. Алгоритмы, вдохновленные природой: учебное пособие / А. П. Карпенко. - 2-е изд. - Москва: Изд-во МГТУ имени М. Э. Баумана, 2017. - 446 с.

8. Королев, С. А. Модификация алгоритма роя частиц на основе метода анализа иерархий / С. А. Королев, Д. В. Майков // Вестник Воронеж. гос. ун-та. Сер. Системный анализ и информационные технологии. - 2019. - № 4. C. 36-46.

9. Sun J., Fang W., Wu X., Palade V., Xu W. Quantum behaved particle swarm optimization: analysis of individual particle behavior and parameter selection // Evolutionary Computation. 2012. Vol. 20, no. 3. P. 349-393. - https://doi. org/10.1162/EVCO_a_00049.

10. Mahdi F. P., Vasant P., Abdullah-AlWadud M., Kallimani V., Watada J. Quantumbehaved bat algorithm for many-objective combined economic emission dispatch problem using cubic criterion function // Neural Computing and Applications. 2019. No. 31. P. 5857-5869. - https://doi.org/10.1007/s00521018-3399-z.

11. Cheng L., Dong H., Zhang Q., Liu Z. ABC algorithm with bees having quantum behavior for constrained optimization // International journal of service and computing oriented manufacturing. 2016. Vol. 2, no. 1. P. 50-66. https://doi.org/10.1504/ijscom.2016.075405.

12. Dahi Z. A., Mezioud C., Draa A. A quantum-inspired genetic algorithm for solving the antenna positioning problem // Swarm and evolutionary computation. 2016. No. 31. P. 2463, doi: 10.1016/j.swevo.2016.06.003. 
13. Saaty T. L. The analytic hierarchy and analytic network measurement processes: applications to decisions under risk // European Journal of Pure and Applied Mathematics. 2008. No. 1. - P. 122-196.

14. Mohazzabi P, Connolly M. J. An algorithm for generating random numbers with normal distribution // Journal of Applied Mathematics and Physics. 2019. Vol. 7, no 11. P. 2712-2722. https://doi.org/10.4236/jamp.2019.711185.

15. Савин, А. В. Байесовский подход в современном анализе: алгоритмы и синтез / A. В. Савин // XXI Междунар. конф. по мягким вычислениям и измерениям (SCM'2018): сб. тр. Междунар. конф. (Санкт-Петербург, 23-25 мая 2018 г.). - СПб., 2018. - С. 635-638.

Королев Станислав Анатольевич - канд. физ.-мат. наук, доцент кафедры «Математическое обеспечение информационных систем» ФГБОУ ВО Ижевский государственный технический университет имени М. Т. Калашникова.

E-mail: stkj@mail.ru

ORCID iD: https://orcid.org/0000-0002-8399-1385

Майков Дмитрий Владимирович - преподаватель математики БПОУ УР Ижевский торговоэкономический техникум.

E-mail: MaykovD@yandex.ru

ORCID iD: https://orcid.org/0000-0002-8198-742X

DOI: https://doi.org/10.17308/sait.2020.2/2914

Received 05.06.2020

ISSN 1995-5499

Accepted 15.06.2020

\title{
QUANTUM MODIFICATION OF THE FRESHWATER HYDRA ALGORITHM FOR SOLVING THE OPTIMIZATION PROBLEM
}

\author{
(C) 2020 S. A. Korolev', D. V. Maikov $₫ 2$ \\ ${ }^{1}$ M. T. Kalashnikov Izhevsk State Technical University \\ 7, Studencheskaya street, 426069 Izhevsk, Russian Federation \\ ${ }^{2}$ Izhevsk Trade and Economic College \\ 20a, Voroshilova street, 426000 Izhevsk, Russian Federation
}

\begin{abstract}
Annotation. One of the stages of solving a number of mathematical modelling problems is finding a point where some function of several variables reaches the largest or the least value. This function is usually high-dimensional and has many local extrema. Such a problem can be successfully solved using population optimisation algorithms, for example, the particle swarm algorithm, the freshwater hydra algorithm, and others. The aim of this work was to improve the freshwater hydra algorithm (H-algorithm) taking into account the quantum mechanics approach. The developed quantum modifications of this algorithm are based on the Analytic Hierarchy Process $(\mathrm{QH}-\mathrm{AHP})$ and the Bayesian approach $(\mathrm{QH}-\mathrm{B})$. The convergence rate of quantum modifications is higher than the convergence rate of the original $\mathrm{H}$-algorithm due to the fact that the position of individuals is determined directly without manipulating their speeds (according to the concepts of quantum mechanics). As a result, during one iteration, individuals can move over a considerable distance, which increases the range of the search space. In addition, this approach allows the individuals to overcome the domains of attraction of local extrema, which prevents the premature algorithm convergence. Optimum values of the parameters of the developed algorithms were found as a result of solving the problem of meta-optimisation. The conversion
\end{abstract}

Maikov Dmitriy V.

e-mail: MaykovD@yandex.ru 
rates of the suggested algorithms of optimisation were compared through the example of various multiextremal test functions, such as the Rosenbrock, Davis, Ackley, and Rastrigin functions. The suggested quantum modifications of the $\mathrm{H}$-algorithm ( $\mathrm{QH}-\mathrm{AHP}$ and $\mathrm{QH}-\mathrm{B}$ ) for various test functions showed different, but on average similar, convergence rates. The convergence rate of quantum modifications of algorithms for various test functions is higher than the convergence rate of the original freshwater hydra algorithm and particle swarm optimisation algorithm. The developed algorithms for solving the optimisation problem can be used in training neural networks, in mathematical modelling of processes and systems in various subject areas at the stage of identifying model parameters, optimisation of characteristics, and the creation of optimal control for such systems, etc..

Keywords: optimisation problem, particle swarm optimisation algorithm, quantum freshwater hydra algorithm, analytic hierarchy process, Bayesian approach, meta-optimisation problem.

\section{CONFLICT OF INTEREST}

The authors declare the absence of obvious and potential conflicts of interest related to the publication of this article.

\section{REFERENCES}

1. Akinshin O. N., Yesikov D. O., Akinshina N. Yu. Peculiarities of solving the problem of enterprise investment portfolio optimization by method of swarm of particles. Izvestiya Tula State University. Technical sciences. 2016. 5. P. 109116. (in Russian)

2. Cheng L., Dong H., Zhang Q., Liu, Z. ABC algorithm with bees having quantum behavior for constrained optimization. International journal of service and computing oriented manufacturing. 2016. 2(1). P. 50-66. Available at: https:// doi.org/10.1504/ijscom.2016.075405

3. Dahi Z. A., Mezioud C., Draa A. A quantum-inspired genetic algorithm for solving the antenna positioning problem. Swarm and evolutionary computation. 2016. (31). P. 24-63. Available at: doi: 10.1016/j.swevo.2016.06.003

4. Ermakov B. S. Optimizatsiya roem chastits v obuchenii iskusstvennykh neironnykh setei [Particle swarm optimization in training artificial neural networks]. Sistemnyi analiz i logistika. 2017. 1. P. 3-9. (in Russian)

5. Esikov O. V., Tsybin S. M., Chernyshkov A. I. Application of the method of Particle Swarm to solve the problem of the distribution of the computational resources on-Board information and control systems according to the criterion of balanced load. Izvestiya Tula State University. Technical sciences. 2017. 9-1. P. 74-81. (in Russian)
6. Gurov E. V., Kovalev V. V., Uvaysova A. S., Uvaysova S. S. Optimization of IIR filters parameters with the use of swarm robotics search algorithm. Innovatsionnye, informatsionnye i kommunikatsionnye tekhnologii. 2017. 1. P. 552-553. (in Russian)

7. Karpenko A. P. Sovremennye algoritmy poiskovoy optimizacii. Algoritmy, vdohnovlennye prirodoi [Modern search engine optimization algorithms. Algorithms inspired by nature]. Moscow, Izdatelstvo moskovskogo gosudarstvennogo tehnicheskogo universiteta imeni N. E. Baumana, 2017. (in Russian)

8. Korolev S. A., Maykov D. V. Identification of a mathematical model and research of the various modes of methanogenesis in mesophilic environments. Computer Research and Modeling. 2012. 4(1). P. 131-141. (in Russian). Available at: doi: 10.20537/2076-7633-2012-4-1-131-141.

9. Korolev S. A., Maykov D. V. Modification of particle swarm algorithm based on hierarchy analysis method. Proceedings of Voronezh State University. Series: Systems analysis and information technologies. 2019. 4. P. 36-46. (in Russian)

10. Korolev S. A., Maykov D. V. Solution of the problem of optimal control of the process of methanogenesis based on the Pontryagin maximum principle. Computer Research and Modeling. 2020. 12 (2). P. 357-367. Available at: doi: 10.20537/2076-7633-2020-12-2-357-367.

11. Mahdi F. P., Vasant P., Abdullah-AlWadud M., Kallimani V., Watada J. Quantum-behaved bat algorithm for many-objective combined economic emission dispatch problem using cubic criterion function. Neural Computing and Applications. 2019. 31. P. 5857-5869. Available at: https://doi.org/10.1007/s00521-018-3399-z. 
12. Mohazzabi P., Connolly M. J. An algorithm for generating random numbers with normal distribution. Journal of Applied Mathematics and Physics. 2019. 7 (11). P. 2712-2722. Available at: https://doi.org/10.4236/jamp.2019.711185.

13. Saaty T. L. The analytic hierarchy and analytic network measurement processes: applications to decisions under risk. European Journal of Pure and Applied Mathematics. 2008. (1). P. 122-196.

14. Savin A. V. Baiesovskii podkhod v sovremennom analize: algoritmy i sintez [Bayesian approach in modern analysis: algorithms and synthesis]. In: XXI International Conference on Soft Computing and Measurement (SCM'2018), 23-25 May 2018, Saint Petersburg, Russia. Saint Petersburg Electrotechnical University "LETI", 2018. P. 635-638. (in Russian)

15. Sun J., Fang W., Wu X., Palade V., Xu W. Quantum behaved particle swarm optimization: analysis of individual particle behavior and parameter selection. Evolutionary Computation. 2012. 20 (3). P. 349-393. Available at: https://doi. org/10.1162/EVCO_a_00049.

Korolev Stanislav A. - PhD in Physics and Mathematics, Associate Professor, Department of Mathematical Support for Information Systems, M.T. Kalashnikov Izhevsk State Technical University. E-mail: stkj@mail.ru

ORCID iD: https://orcid.org/0000-0002-8399-1385

Maikov Dmitriy V. - teacher of mathematics, Izhevsk Trade and Economics College.

E-mail: MaykovD@yandex.ru

ORCID iD: https://orcid.org/0000-0002-8198-742X 